\title{
Recurrent vaginal intraepithelial neoplasia successfully treated with topical imiquimod: A case report
}

\author{
NANAYO SASAGASAKO ${ }^{1}$, KENZO KOSAKA $^{1}$, YUSUKE SAGAE ${ }^{1}$, KUNITOSHI ITOH $^{1}$, \\ JUNICHI ARATAKE ${ }^{1}$, KAORI YAMADA ${ }^{1}$, YOSHIHIDE INAYAMA ${ }^{1}$, REI GOU $^{1}$, ATSUKO KAWAMURA ${ }^{1}$, \\ MEGUMI YAMANISHI $^{1}$, YUKIO YAMANISHI ${ }^{1}$, MAKOTO SUZUKI ${ }^{2}$ and KAZUMORI ARAI ${ }^{2}$ \\ Departments of ${ }^{1}$ Obstetrics and Gynecology, and ${ }^{2}$ Pathology, Shizuoka General Hospital, Shizuoka 420-8527, Japan
}

Received December 9, 2019; Accepted May 22, 2020

DOI: $10.3892 / \mathrm{mco} .2020 .2089$

\begin{abstract}
Vaginal intraepithelial neoplasia (VAIN) is a rare disease associated with human papillomavirus infection. High-grade VAIN is typically treated with either excisional or ablative therapy. However, recurrent VAIN lesions are common and these treatments cause vaginal scarring. Recent studies have indicated that $5 \%$ imiquimod is an effective treatment for VAIN. The present report describes a case of a woman diagnosed with recurrent VAIN 3 who was treated with a 5\% topical imiquimod cream and achieved a complete response after excision and $\mathrm{CO}_{2}$ laser vaporization. A 53-year-old, gravida 5, para 2 postmenopausal woman who was diagnosed with papillary squamous cell carcinoma by biopsy underwent conization, total abdominal hysterectomy and bilateral salpingo-oophorectomy. A histological examination revealed grade 3 cervical intraepithelial neoplasia with free surgical margins. At 3 years after the hysterectomy, the vaginal smear revealed atypical squamous cells, leading to a pathological diagnosis of VAIN 3. Partial vaginectomy was performed, and VAIN 3 was detected in the lesion with positive margins. At 4 months into follow-up, the vaginal smear revealed a high-grade squamous intraepithelial lesion (HSIL), and subsequent biopsy during colposcopy revealed a pathological diagnosis of VAIN 3. At 3 months after $\mathrm{CO}_{2}$ laser vaporization, the vaginal smear revealed HSIL with suspected recurrence and imiquimod treatment was initiated. One sachet of $5 \%$ imiquimod cream $(0.25 \mathrm{~g})$ was placed in the entire vagina three times per week for 14 weeks with no apparent complications. At 3 years after the treatment, there has been no recurrence. This case demonstrated that topical imiquimod with careful follow-up is an effective treatment for VAIN and is well-tolerated. Further clinical evidence of the effectiveness
\end{abstract}

Correspondence to: Dr Nanayo Sasagasako, Department of Obstetrics and Gynecology, Shizuoka General Hospital, 27-1 Kita-Ando 4-chome, Aoi-ku, Shizuoka 420-8527, Japan

E-mail: ynanayo@kuhp.kyoto-u.ac.jp

Key words: vaginal intraepithelial neoplasia, imiquimod, non-surgical treatment, recurrence, side effects and safety of imiquimod in patients diagnosed with VAIN is required.

\section{Introduction}

Vaginal intraepithelial neoplasia (VAIN) is a rare disease associated with human papillomavirus (HPV) infection. The incidence of VAIN has been reported at approximately $0.2-0.3$ per 100,000 women and is considered to be a precursor of vaginal carcinoma (1). In the World Health Organization (WHO) 2014 classification, VAIN lesions are graded as vaginal low-grade and high-grade squamous intraepithelial lesions (LSIL and HSIL, respectively) with LSIL including VAIN 1 and HSIL including VAIN 2 and VAIN 3. VAIN 1 can also be considered a form of productive HPV infection, which can be expectantly managed with a spontaneous regression rate of over $50 \%$ (2). VAIN 2 and 3 are considered precancerous lesions, so treatment is required for high-grade VAIN. Especially, VAIN 3 owes to the high risk of progression to carcinoma in approximately $11-13 \%$ of cases (3). However, current treatment recommendations vary, and there is no universally accepted standard of care as the best treatment modality (4).

High-grade VAIN is usually treated with either excisional or ablative therapy, however recurrent VAIN lesions are common and require repeat treatments which may be mutilating and cause vaginal scarring (3). Imiquimod is a toll-like receptor 7 (TLR7) agonist which modulates an immune response by inducing secretion of interferon- $\alpha$ and interferon- $\gamma$, and is used for the treatment of condylomas and other HPV-related neoplasias including VAIN (5). Recently some studies have reported that $5 \%$ imiquimod is an effective treatment for VAIN $(5,6)$.

We here report a case of a woman who was diagnosed with recurrent VAIN 3 and treated with 5\% topical imiquimod cream after surgical treatment and achieved a complete response. This case thus provides further evidence for the clinical efficacy and safety of 5\% topical imiquimod in the treatment of recurrent VAIN.

\section{Case report}

A 53-year-old, gravida 5, para 2 postmenopausal woman with no past medical history was diagnosed with papillary 
squamous cell carcinoma by biopsy (Fig. 1) and underwent conization, resulting in a pathological diagnosis of grade 3 cervical intraepithelial neoplasia (CIN). Total abdominal hysterectomy and bilateral salpingo-oophorectomy were performed and a histological examination revealed CIN 3 with free surgical margins.

Eight months after the hysterectomy, a vaginal smear revealed atypical squamous cells, cannot exclude HSIL (ASC-H). Because no abnormal mitosis was observed in the biopsy, p16 was negative and Mib-1 index was 0.1-1\%, atrophy or maturation arrest of squamous epithelia was suspected. Cytology further revealed ASC-H in succession with no apparent finding related to invasive carcinoma; therefore, careful follow-up was decided with no further treatment.

Three years after the hysterectomy, a follow-up vaginal smear revealed ASC-H, and vaginoscopy after the application of acetic acid showed increased vascularity; therefore, biopsy was performed. On microscopic examination, atypia was observed in all layers of the epithelium with no interstitial infiltration, leading to a pathological diagnosis of VAIN 3. No lesion was detected on contrast magnetic resonance imaging (Fig. 2).

Partial vaginectomy with a loop electrosurgical excision procedure (LEEP) was performed under spinal anesthesia. However, it was difficult to perform the appropriate peeling operation on the right and left sides of the stump, and a lesion at the 3-o'clock position remained. The lesion was additionally peeled. The resected specimen was $5.4 \times 3.8 \mathrm{~cm}$ in size. Microscopic examination demonstrated a VAIN 3 lesion measuring $3.5 \times 2.5 \mathrm{~cm}$ within the specimen. Neoplastic cells were confined to the epithelium, but the surgical margin was positive. There were also neoplastic cells in the additionally resected specimen. Neither postoperative radiation therapy nor chemotherapy was planned, opting instead for careful follow-up.

Four months after the vaginectomy, a vaginal smear revealed HSIL. Vaginal biopsy was performed 1 month later on the lesion at the 8-o'clock position during colposcopy, resulting in a pathological diagnosis of VAIN 3 (Fig. 3). Eight months after the vaginectomy, $\mathrm{CO}_{2}$ laser vaporization was performed under spinal anesthesia. A small mass at the 7 o'clock position of the vaginal wall was resected using a scalpel. Microscopic examination demonstrated atypia in almost all layers of the epithelium, and the pathological diagnosis was VAIN 3 with a positive margin.

Three months after $\mathrm{CO}_{2}$ laser excision, the vaginal smear revealed HSIL with suspected recurrence. Imiquimod treatment was then initiated by placing a sachet of $5 \%$ imiquimod cream $(0.25 \mathrm{~g})$ in the entire vagina three times weekly. The institutional review board approved the use of imiquimod for VAIN (SGHIRB\#2019049). As imiquimod is not a standard treatment for VAIN, follow-up vaginal smear was conducted monthly after the start of imiquimod treatment to evaluate the efficacy of the treatment. At 53 days and 74 days after the treatment, the vaginal smear revealed ASC-H and was negative for intraepithelial lesion or malignancy (NILM). A vaginal smear 1 month later further confirmed the NILM status (Table I). The treatment was continued for 14 weeks with no apparent complication. As of 3 years after the treatment, there has been no recurrence.
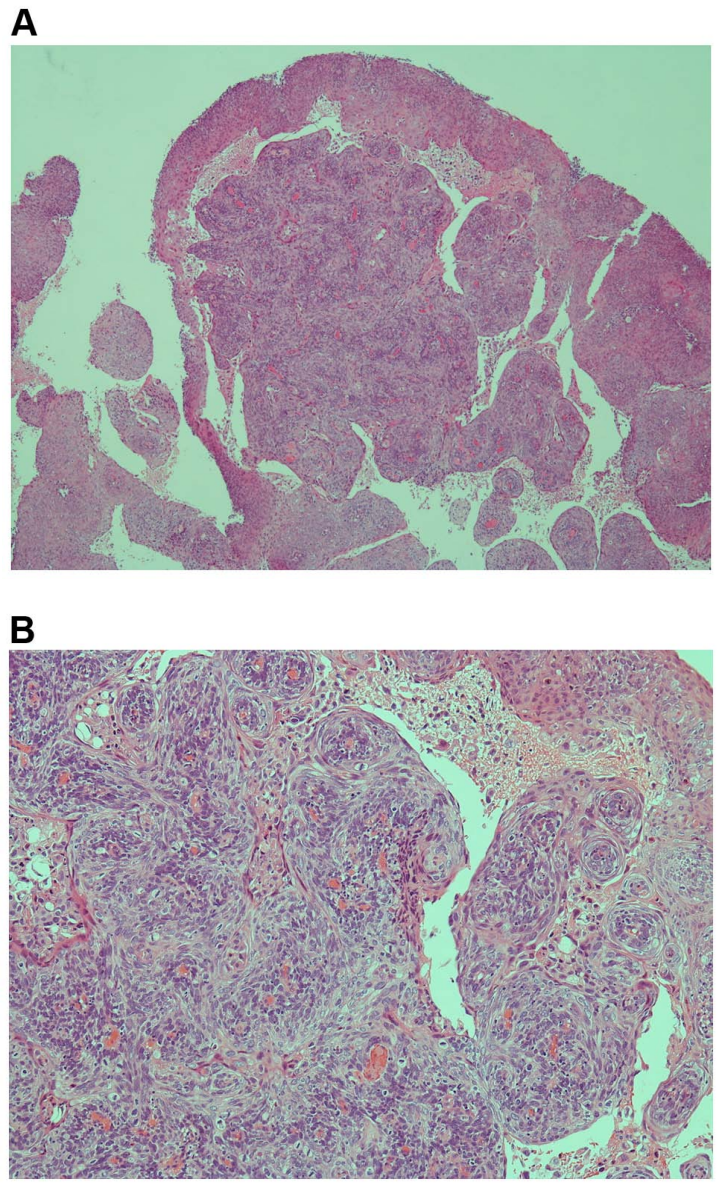

Figure 1. Biopsy of the cervical tumor. Papillary squamous cell carcinoma was observed. (A) Hematoxylin and eosin staining. Magnification, x4 (B) Hematoxylin and eosin staining. Magnification, x10.

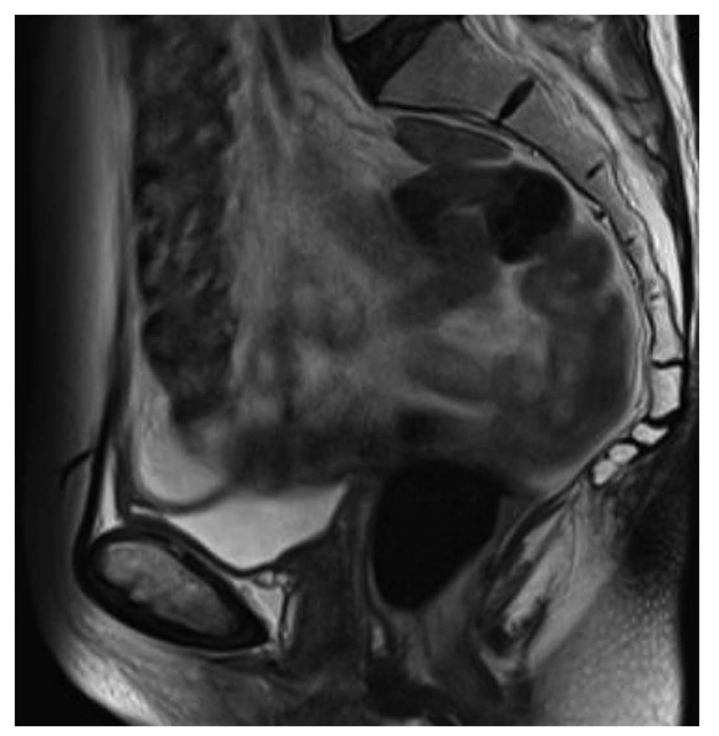

Figure 2. Sagittal T2-weighted MRI imaging of the pelvis. No lesion was identified.

\section{Discussion}

Surgical treatment modalities for high-grade VAIN include partial vaginectomy, wide local excision, a LEEP, and $\mathrm{CO}_{2}$ 
Table I. Results of pathological examination.

\begin{tabular}{|c|c|c|}
\hline Months post-operation & Intervention/specimen & Result \\
\hline 0 & $\mathrm{TAH}+\mathrm{BSO}$ & CIN 3, cut end negative \\
\hline 2 & Cytology & NILM \\
\hline 4 & Cytology & ASC-US \\
\hline 6 & Cytology & NILM \\
\hline 8 & Cytology & ASC-H \\
\hline 10 & Vaginal biopsy & Atypical squamous epithelia ${ }^{a}$ \\
\hline $12,15,18$ & Cytology & ASC-H \\
\hline 22 & Cytology & NILM \\
\hline $25,29,33$ & Cytology & ASC-H \\
\hline 36 & Biopsy & VAIN 3 \\
\hline 39 & Partial vaginectomy by LEEP & VAIN 3, cut end positive \\
\hline 40 & Cytology & NILM \\
\hline 41 & Cytology & SCC \\
\hline 43 & Cytology & HSIL \\
\hline 45 & Vaginal biopsy & VAIN 3 \\
\hline 47 & $\mathrm{CO} 2$ laser vaporization, resection & VAIN 3, cut end positive \\
\hline 48 & Cytology & NILM \\
\hline 48 & Cytology & ASC-H \\
\hline 50 & Cytology & HSIL \\
\hline 51 & \multicolumn{2}{|c|}{ Administration of $5 \%$ imiquimod cream } \\
\hline 51 & Cytology & ASC-H \\
\hline $52-90$ & Cytology & NILM \\
\hline
\end{tabular}

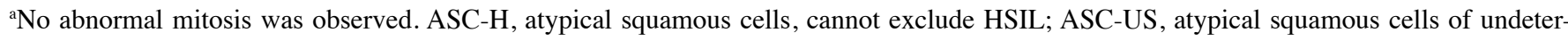
mined significance; CIN, cervical intraepithelial neoplasia; HSIL, high-grade squamous intraepithelial lesion; LEEP, loop electrosurgical excision procedure; NILM, negative for intraepithelial lesion or malignancy; SCC, squamous cell carcinoma; TAH + BSO, total abdominal hysterectomy + bilateral salpingo-oophorectomy.

A

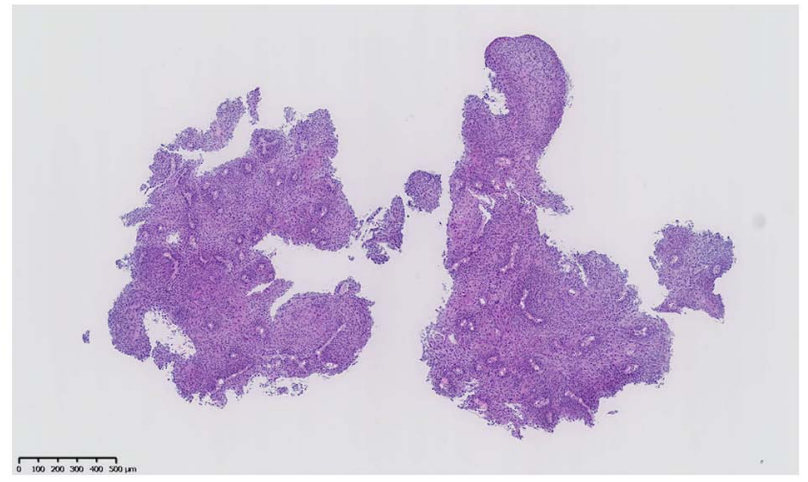

B

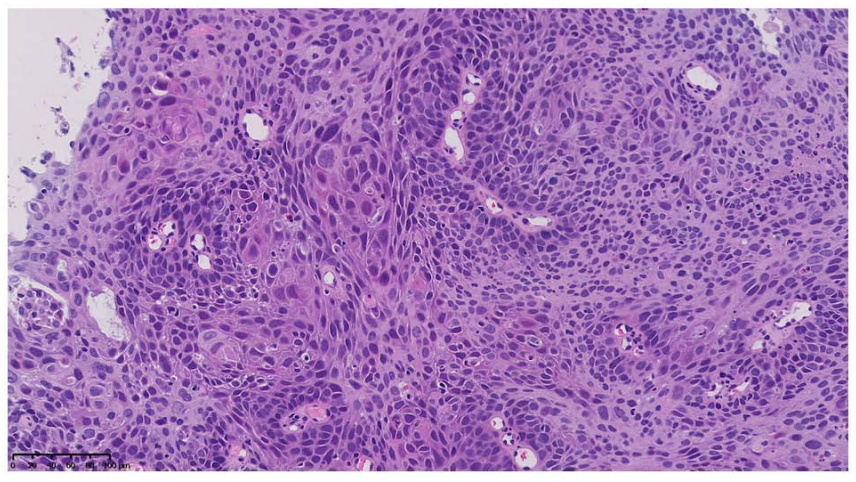

Figure 3. Vaginal biopsy revealing atypical cells of vaginal intraepithelial neoplasia 3. (A) Hematoxylin and eosin staining in a low magnification image. Scale bar, $500 \mu \mathrm{m}$. (B) Hematoxylin and eosin staining in a high magnification image. Scale bar, $100 \mu \mathrm{m}$.

laser vaporization. High-grade VAIN is typically treated with either excisional or ablative therapy; however, the VAIN lesions often recur, requiring repeated treatments that may cause severe damage to the tissue and vaginal scarring (3). Moreover, surgical treatments require anesthesia and can cause complications such as bleeding, infection, scarring, and injury of neighboring anatomical structures as well as high recurrence rates (4). Therefore, alternative treatments are desired.
Non-surgical treatment modalities include imiquimod, 5-fluorouracil, and vaginal estrogens as well as radiotherapy (4). Imiquimod is a TLR7 agonist, which is used for the treatment of condylomas and other HPV-related neoplasias, including VAIN. Imiquimod triggers the innate immune response via TLR7-Myd88-dependent signaling by interacting with TLR7 that is expressed by myeloid dendritic cells (DCs), plasmacytoid DCs, monocytes, and macrophages. Subsequently, TLR7 
activation induces the secretion of various pro-inflammatory cytokines, such as interferon- $\alpha$ and interferon- $\gamma$, to in turn induce a Th1 response leading to the apoptosis of cancer cells $(4,7)$. Imiquimod has been shown to promote histological clearance of basal cell carcinoma (BCC) in clinical studies and is approved for the treatment of BCC by Food and Drug Administration $(8,9)$. Moreover, imiquimod has been used in combination with chemotherapy to treat breast cancer patients in a phase II clinical trial (10). Several studies reported promising results with the use of imiquimod for the treatment of patients with vulvar intra-epithelial neoplasia (VIN), CIN, and VAIN (5,6,11-14). A meta-analysis including 94 patients with VAIN treated with 5\% imiquimod showed a complete response, HPV clearance, and non-recurrence in 76.5, 52.5, and $94.3 \%$ of patients, respectively (4).

Imiquimod has emerged as a potentially useful treatment for CIN, VIN, and VAIN; however, further clinical data are required to evaluate the safety profile. Some physicians reported systemic and local adverse effects after imiquimod treatment for CIN, including headache, fever, fatigue, myalgia, vaginal discharge, vaginal bleeding, vulvar pruritus, vulvar pain, and vaginal edema (15). Wouters et al (16) reported serious adverse events in three patients who received imiquimod intravaginally for CIN: Two of the patients were hospitalized with headache, nausea, diarrhea, malaise, leukopenia, and hyponatremia, and the other patient exhibited systemic symptoms and erosion of the cornea. Although none of these adverse effects was directly proven to be caused by imiquimod, all of the symptoms disappeared after discontinuation of imiquimod. Such systemic adverse effects could be caused by the imiquimod-induced peripheral Th1 immune response (15). Tainio et al (3) reported that all 10 patients with VAIN, who received imiquimod in a randomized control study developed side effects such as flu-like symptoms, local irritation in the vagina and vulva, and lower abdominal pain. In one patient, the dosage was reduced by half, but no patient required discontinuation of the treatment. Moreover, one study concluded that non-steroidal anti-inflammatory drugs can safely be used to reduce side effects in usual type VIN (17). The same could apply to VAIN. Overall, the side effects of imiquimod are considered to be well-tolerated, and patients can be treated through outpatient management. However, clinicians prescribing this treatment should monitor patients closely for potential severe side effects.

In the present case, a patient with recurrence of VAIN after surgical treatments received imiquimod with no apparent adverse events, and the lesion ultimately disappeared. This case therefore provides further support that topical imiquimod with careful follow-up is effective and well-tolerated for the treatment of recurrent VAIN. Nevertheless, more studies should be accumulated on the clinical experience with imiquimod in patients with VAIN. In conclusion, imiquimod can be considered as one of the options for treatment of refractory VAIN.

\section{Acknowledgements}

Not applicable.

\section{Funding}

No funding was received.

\section{Availability of data and materials}

The datasets used and/or analyzed during the current study are available from the author on reasonable request.

\section{Authors' contributions}

NS, KK and YI interpreted the case and reviewed the patient's clinical information, and drafted the manuscript and revised it critically for important intellectual content. YS and KI were responsible for the patient's treatment and management decisions. JA, KY, RG, AK, MY and YY revised the manuscript critically for important intellectual content. MS and KA were responsible for the pathological diagnosis. All authors read and approved the final manuscript.

\section{Ethics approval and consent to participate}

The Institutional Review Board of Shizuoka General Hospital approved the use of imiquimod for VAIN (SGHIRB\#2019049).

\section{Patient consent for publication}

Informed consent for publication was obtained from the patient.

\section{Competing interests}

The authors declare that they have no competing interests.

\section{References}

1. De Vuyst H, Clifford GM, Nascimento MC, Madeleine MM and Franceschi S: Prevalence and type distribution of human papillomavirus in carcinoma and intraepithelial neoplasia of the vulva, vagina and anus: A meta-analysis. Int J Cancer 124: 1626-1636, 2009.

2. Aho M, Vesterinen E, Meyer B, Purola E and Paavonen J: Natural history of vaginal intraepithelial neoplasia. Cancer 68: 195-197, 1991.

3. Tainio K, Jakobsson M, Louvanto K, Kalliala I, Paavonen J, Nieminen P and Riska A: Randomised trial on treatment of vaginal intraepithelial neoplasia-Imiquimod, laser vaporisation and expectant management. Int J Cancer 139: 2353-2358, 2016.

4. Tranoulis A, Laios A, Mitsopoulos V, Lutchman-Singh K and Thomakos N: Efficacy of 5\% imiquimod for the treatment of Vaginal intraepithelial neoplasia-A systematic review of the literature and a meta-analysis. Eur J Obstet Gynecol Reprod Biol 218: 129-136, 2017.

5. De Witte CJ, Van De Sande AJM, Van Beekhuizen HJ, Koeneman MM, Kruse AJ and Gerestein CG: Imiquimod in cervical, vaginal and vulvar intraepithelial neoplasia: A review. Gynecol Oncol 139: 377-384, 2015.

6. Policiano ACF, Lopes JPM, Barata SAM, Colaço AM and Calhaz-Jorge C: Topical therapy with imiquimod for vaginal intraepithelial neoplasia: A Case Series. J Low Genit Tract Dis 20: e34-e36, 2016.

7. Chi H, Li C, Zhao FS, Zhang L, Ng TB, Jin G and Sha O: Anti-tumor activity of toll-like receptor 7 agonists. Front Pharmacol 8: 304, 2017.

8. Geisse J, Caro I, Lindholm J, Golitz L, Stampone P and Owens M: Imiquimod 5\% cream for the treatment of superficial basal cell carcinoma: Results from two phase III, randomized, vehicle-controlled studies. J Am Acad Dermatol 50: 722-733, 2004.

9. Hanna E, Abadi R and Abbas O: Imiquimod in dermatology: An overview. Int J Dermatol 55: 831-44, 2016.

10. Salazar LG, Lu H, Reichow JL, Childs JS, Coveler AL, Higgins DM, Waisman J, Allison KH, Dang Y and Disis ML: Topical imiquimod plus nab-paclitaxel for breast cancer cutaneous metastases. JAMA Oncol 3: 969, 2017. 
11. Terlou A, van Seters M, Ewing PC, Aaronson NK, Gundy CM, Heijmans-Antonissen C, Quint WGV, Blok LJ, van Beurden M and Helmerhorst TJM: Treatment of vulvar intraepithelial neoplasia with topical imiquimod: Seven years median follow-up of a randomized clinical trial. Gynecol Oncol 121: 157-162, 2011.

12. Buck HW and Guth KJ: Treatment of vaginal intraepithelial neoplasia (primarily low grade) with imiquimod $5 \%$ cream. J Low Genit Tract Dis 7: 290-293, 2003.

13. Diaz-Arrastia C, Arany I, Robazetti SC, Dinh TV, Gatalica Z, Tyring SK and Hannigan E: Clinical and molecular responses in high-grade intraepithelial neoplasia treated with topical imiquimod 5\%. Clin Cancer Res 7: 3031-3033, 2001.

14. Haidopoulos D, Diakomanolis E, Rodolakis A, Voulgaris Z, Vlachos $\mathrm{G}$ and Intsaklis A: Can local application of imiquimod cream be an alternative mode of therapy for patients with high-grade intraepithelial lesions of the vagina? Int J Gynecol Cancer 15: 898-902, 2005.

15. Koeneman MM, van de Sande AJ, van Beekhuizen HJ, Gerestein KG, van de Laar R, Kruitwagen RF and Kruse AJ: Physicians' awareness, attitudes, and experiences regarding imiquimod treatment of vaginal and cervical intraepithelial neoplasia. J Low Genit Tract Dis 20: 75-79, 2016.
16. Wouters T, Hendriks N, Koeneman M, Kruse AJ, van de Sande A van Beekhuizen HJ, Gerestein KG, Bekkers RLM and Piek JMJ: Systemic adverse events in imiquimod use for cervical intraepithelial neoplasia - A case series. Case Reports Women's Heal 21: e00105, 2019.

17. Terlou A, Kleinjan A, Beckmann I, Heijmans-Antonissen C, van Seters M, Santegoets LAM, van Beurden M, Helmerhorst TJM and Blok LJ: Nonsteroidal anti-inflammatory drugs do not interfere with imiquimod treatment for usual type vulvar intraepithelial neoplasia. Int J Cancer 128: 2463-2469, 2011. 